\title{
Rheological Behavior of Modified Starch/EVA Blends
}

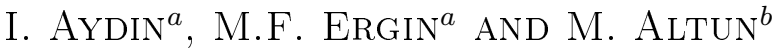 \\ ${ }^{a}$ Rheology Laboratory, Chemical Engineering Department, Faculty of Engineering, Istanbul University \\ Avcilar Campus, Avcilar 34320, Istanbul, Turkey \\ ${ }^{b}$ Food Laboratory, Chemistry Department, Faculty of Engineering, Istanbul University \\ Avcilar Campus, Avcilar 34320, Istanbul, Turkey
}

\begin{abstract}
There is a current research tendency towards the biodegradable thermoplastic materials from renewable resources since the environmental restraints play vital role in recent years. Starch is one of the renewable resources and abundantly available in the form of plant products. It is possible to blend starch with various thermoplastic materials to achieve such aims. In this study, an ethylene-vinyl acetate (EVA) copolymer resin (Luvax $40 \mathrm{~W}$, DuPont) was blended with a modified starch (Maize Starch, Pendik Nisasta) in various amounts by means of a single screw extruder with different screw speeds. For this purpose, first starch was modified in a four neck spherical glass reactor. Modified starch was then blended with the EVA copolymer resin. Rheological analyses of these composites were performed by means of an AR G2 rheometer (TA Instruments) with parallel-plate geometry in a steady state condition. Rheological behavior and properties were recorded. The changes in viscosity with different temperatures, stress and strain dependence as well as general constitutive material behavior were determined in detail. As a result, the best formulation and process conditions were determined for the reasonable processability of starch/EVA blends from the obtained rheological data.
\end{abstract}

DOI: $10.12693 /$ APhysPolA.125.503

PACS: 83.85.Cg, 83.50.Xa, 83.80.Mc, 83.80.Tc

\section{Introduction}

The disposal of thermoplastic products is now one of the main concerns of our developing century. Degradation is a disposal method, which is highly justified in many thermoplastic applications. Current researches are focusing upon controlling the factors that affect these degradations since there is a need to develop new biodegradable materials as well as biodegrade existing plastic materials as the thermoplastic solid waste increases especially in daily life in urban areas.

Ethylene-vinyl acetate (EVA) copolymer resin with its many kinds is a commodity plastic that is extensively used in industries where adhesives are used and other applications. Blending process of this material, with the best formulation and process conditions, is important to obtain required mechanical properties. An extruder is used in this study to blend EVA resin with other biodegradable additive. Biodegradation is the chemical breakdown of materials by a physiological environment in which organic matter is converted into minerals. Some additives consumed by microorganisms enhance the biodegradation process.

EVA is photodegradable and starch is biodegradable. Starch is one of the renewable resources and abundantly available in the form of plant product such as maize, corn, and potato. This photo- and biodegradable starch filled EVA products are used in many industries manufactured by means of either by extrusion thermoforming or injection molding techniques. In this study, EVA was melt blended with modified starch at different concentrations of EVA using a single screw extruder with a transparent barrel. The influence of three different concentrations $(5,10$, and $15 \%)$ of the EVA on the rheological properties was evaluated.

\section{Experimental}

\subsection{Materials}

Ethylene-vinyl acetate was used as matrix polymer for the materials which were investigated in this study. The EVA was acquired from the DuPont (Belgium). Table I shows properties of the EVA according to the Material Data Sheet. The starch (maize starch) was supplied by Pendik Nisasta, Turkey. Modified starch which was used in this study was prepared in our laboratory.

TABLE I

Some properties of EVA according to the Material Data Sheet.

\begin{tabular}{c|c|c|c}
\hline \hline Property & Unit & Value & Test method \\
\hline density & $\mathrm{g} / \mathrm{cm}^{3}$ & 0.965 & ASTM D792 \\
melt flow rate & $\mathrm{g} / 10 \mathrm{~min}$ & 52 & ASTM D1238 \\
melting point (DSC) & ${ }^{\circ} \mathrm{C}$ & 47 & ASTM D3418 \\
maximum processing & ${ }^{\circ} \mathrm{C}$ & 230 & \\
temperature & &
\end{tabular}

Composition of EVA which was used in this study was $40 \%$ by weight vinyl acetate comonomer content.

\subsection{Preparation of EVA-modified starch}

Firstly, modified starch was prepared by dispersing $3 \%(\mathrm{w} / \mathrm{w})$ starch in water. The starch dispersion was heated on a hot plate to $90^{\circ} \mathrm{C}$ and held at that temperature for $20 \mathrm{~min}$. The heated starch solution was cooled to about $65^{\circ} \mathrm{C}$ and the required amount of glycerol was added to the starch solution. The starch solution was then poured onto Teflon-coated glass plates. The cast modified starch was allowed to air dry for about $48 \mathrm{~h}$ before peeling them from the plates [1]. 
EVA-modified starch blend was prepared by mixing modified starch pellets in EVA matrix using melt blending technique. EVA was manually mixed with different concentration of modified starch whose ratio is 5,10 , and $15 \mathrm{wt} \%$. Before the melt blending in a single screw extruder, the modified starch was dried in a conventional oven at $80^{\circ} \mathrm{C}$ for $24 \mathrm{~h}$ in order to reduce the moisture content. Then, the pre-mix was compounded using a special single screw extruder. This was designed for the determination of material flow at each zone which had different screw configurations in order to achieve plastication. The barrel of this extruder used throughout this study is composed of transparent glass which is resistant to thermal shocks.

Hence, rheological flow of studied material and the whole plastication process (including solid conveying, melting and melt conveying) could be clearly observed and the process was enabled to be recorded behind the transparent barrel by means of cameras. The diameter and $L / D$ ratio of this specially designed single screw extruder was $15 \mathrm{~mm}$ and the 29, respectively. The single screw extruder (Fig. 1) was capable of both distributive and dispersive mixing with its composing elements. The extrudate from the outlet was cooled in open-air for further use.

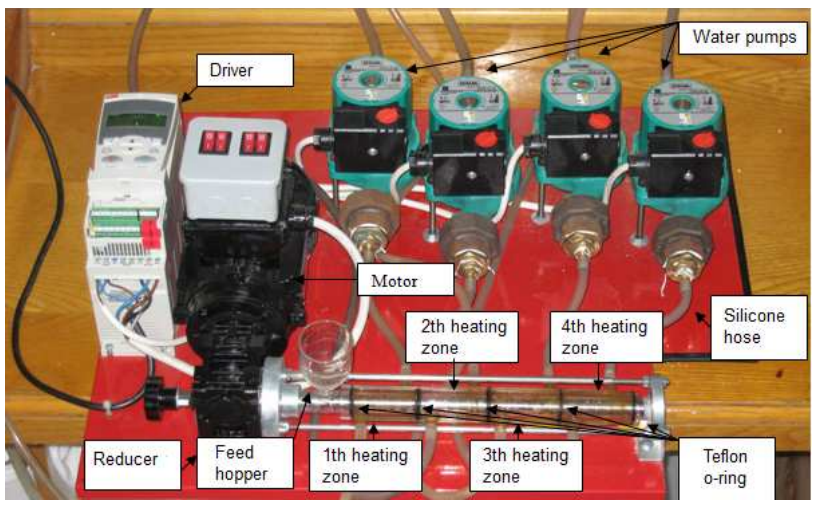

Fig. 1. Extruder with glass barrel.

The processing conditions of single screw extruder are listed in Table II. The samples were referred to as MS-5, MS-10, MS-15 for the corresponding modified starch concentration of 5,10 , and $15 \mathrm{wt} \%$, respectively. The same extrusion procedure was carried out on EVA to compare pure material with its corresponding blend. The extruded pellets obtained from the single screw extruder were cooled as mentioned before, and then pellets were prepared for a Rheometer instrument.

\subsection{Samples characterization}

The melt rheological measurements were carried out using a Rheometer instrument model AR G2 made by TA Instruments, USA (Fig. 2). The complex viscosities of neat EVA and its blend were measured under torsion mode using frequency sweep scheme between parallel
TABLE II

Processing conditions of the single screw extruder.

\begin{tabular}{c|c|c|c|c}
\hline \hline \multicolumn{4}{|c|}{ Barrel temperature $\left[{ }^{\circ} \mathrm{C}\right]$} & Screw speed \\
$\begin{array}{c}\text { Feed } \\
\text { zone }\end{array}$ & Zone 2 & Zone 3 & Zone 4 & [rpm] \\
\hline 25 & 85 & 88 & 86 & 400
\end{tabular}

plates fixture with a diameter equal to $25 \mathrm{~mm}$ and a constant gap value of $1 \mathrm{~mm}$. Stress sweep tests were also performed on representative samples to determine their linear viscoelastic region. Prior, frequency sweep tests were performed at a constant temperature of $85^{\circ} \mathrm{C}$. Oscillation stress was kept constant at $200 \mathrm{~Pa}$. This value is within the range of linear viscoelastic region of each blend, which was determined based on the oscillation stress vs. \% (shear) strain curve generated from stress sweep test. The data obtained from this frequency sweep test were storage modulus $\left(G^{\prime}\right)$, loss modulus $\left(G^{\prime \prime}\right)$, and complex viscosity $\left(\eta^{*}\right)$ versus angular frequency.

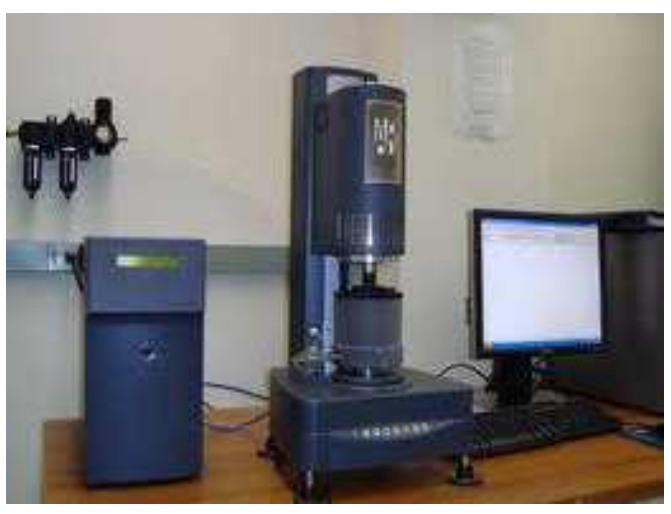

Fig. 2. TA AR G2 Rheometer instrument.

\section{Results and discussion}

Shown in Fig. 3 and Fig. 4 are the results of the frequency sweep tests that were performed. As the frequency increased, the storage modulus $\left(G^{\prime}\right)$ and loss modulus $\left(G^{\prime \prime}\right)$ of EVA-modified starch blends increased. Melt rheological behavior of EVA significantly changed after the incorporation of modified starch into the EVA matrix. At lower frequencies region $(\omega<10 \mathrm{rad} / \mathrm{s})$, the melt rheological behavior of blends was very different from that of the pure EVA especially at higher modified starch concentration (10 and $15 \mathrm{wt} \%$ ). Both $G^{\prime}$ and $G^{\prime \prime}$ for the blends exhibited diminished frequency dependence, while at lower blend concentration (5 wt\%) the melt rheological behavior looked similar to that of pure EVA. This indicates that the viscoelastic properties are still dominated by the polymer matrix. In addition, at lower frequencies region $(\omega>10 \mathrm{rad} / \mathrm{s})$, as modified starch concentration in the EVA matrix increased, viscoelastic response of the modified starch 
gradually changed from pseudoliquid-like $\left(G^{\prime} \backslash G^{\prime \prime}\right)$ to pseudosolid-like $\left(G^{\prime} \backslash G^{\prime \prime}\right)$ behavior. In fact, for EVA-modified starch blend with highest modified starch concentration (MS-15), $G^{\prime}$ was not very sensitive to the increase of angular frequency at lower frequencies region as compared to that of the pure EVA. The $G^{\prime}$ of MS-15 exceeded its $G^{\prime \prime}$ at frequency lower than $0.1 \mathrm{rad} / \mathrm{s}$, which means that characteristic of the material exhibits a pseudosolid-like behavior. Similar results have been observed by Koo et al. [2].

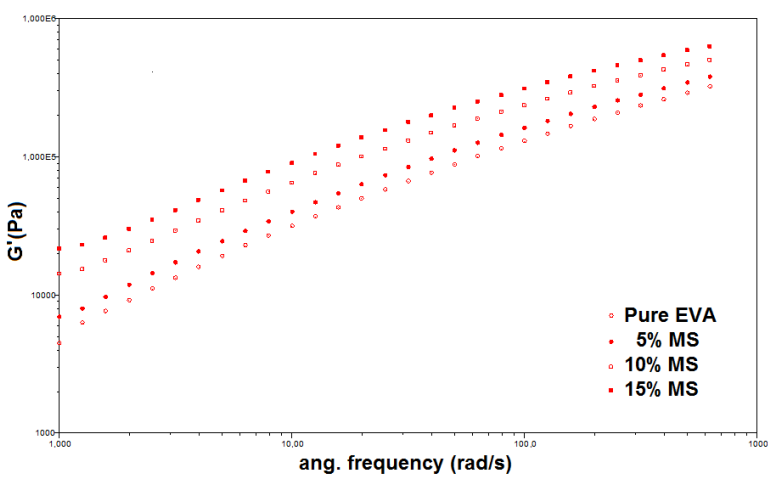

Fig. 3. Storage modulus vs. angular frequency of EVA-modified starch at $85^{\circ} \mathrm{C}$.

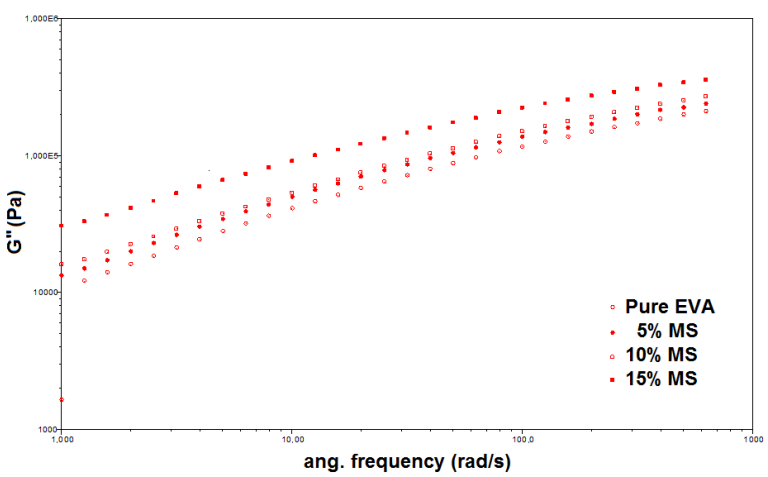

Fig. 4. Loss modulus vs. angular frequency of EVA-modified starch at $85^{\circ} \mathrm{C}$.
It is suggested that the polymer chains could not relax completely due to the interaction between polymer and modified starch. Interfacial characteristics between modified starch particles and EVA matrix as well as the microstructural difference gave a strong relationship between morphological and rheological properties, which manifested in the change of the viscoelastic properties $[2-5]$.

\section{Conclusions}

Melt-blended EVA-modified starch blends with different modified starch concentration were successfully prepared. The effect of modified starch concentration on the rheological properties of the prepared blends has been studied and evaluated. These results suggested that the optimum loading is 5\%. Morphological study revealed that all the samples showed good distribution and dispersion of modified starch particles in EVA matrix at all concentrations.

In rheological behavior, the blends showed higher storage modulus, loss modulus, and complex viscosity than the corresponding pure EVA. Evidence of changes from pseudoliquid-like $\left(G^{\prime} \backslash G^{\prime \prime}\right)$ to pseudosolid-like $\left(G^{\prime} \backslash G^{\prime \prime}\right)$ appeared as the modified starch concentration increased which showed an intense relationship with their morphologies.

\section{References}

[1] N. Reddy, Y. Yang, Food Chemistry 118, 702 (2010).

[2] C.M. Koo, M.J. Kim, M.H. Choi, S.O. Kim, I.J. Cheung, J. Appl. Polym. Sci. 88, 1526 (2003).

[3] H.B. Kim, J.S. Choi, C.H. Lee, S.T. Lim, M.S. John, H.J. Choi, Euro Polym. J. 41, 679 (2005).

[4] Y.T. Lim, O.O. Park, Rheol. Acta 220, 40 (2001).

[5] S.S. Ray, M. Okamoto, Prog. Polym. Sci. 28, 1539 (2003). 